\title{
Multimodal optical imaging of mouse Ischemic cortex
}

\author{
Phillip B Jones $\mathrm{PhD}^{a}$, Hwa Kyoung Shin ${ }^{b}, \mathrm{PhD}$, Andrew K Dunn, $\mathrm{PhD}^{a}$, Bradley T Hyman, \\ $\mathrm{MD}, \mathrm{PhD}^{c}$, David A Boas, $\mathrm{PhD}^{a}$, Michael A Moskowitz, $\mathrm{MD}^{b}$, Cenk Ayata, $\mathrm{MD}^{b}$ \\ ${ }^{a}$ Martinos Center for Biomedical Imaging, \\ ${ }^{b}$ Stroke and Neurovascular Regulation Laboratory, \\ ${ }^{c}$ MassGeneral Institute for Neurodegenerative disorders, \\ ${ }^{d}$ Stroke Service and Neuroscience Intensive Care Unit, Department of Neurology, \\ Massachusetts General, Hospital, Harvard Medical School, Charlestown, MA 02129, USA
}

\begin{abstract}
Real time investigation of cerebral blood flow $(\mathrm{CBF})$, and oxy/deoxy hemoglobin volume (HbO,HbR) dynamics has been difficult until recently due to limited spatial and temporal resolution of techniques like laser Doppler flowmetry and MRI. This is especially true for studies of disease models in small animals, owing to the fine structure of the cerebral vasculature. The combination of laser speckle flowmetry (LSF) and multi-spectral reflectance imaging (MSRI) yields high resolution spatio-temporal maps of hemodynamic changes in response to events such as sensory stimuli or arterial occlusion. Ischemia was induced by distal occlusion of the medial cerebral artery (dMCAO). Rapid changes in $\mathrm{CBF}, \mathrm{HbO}$, and $\mathrm{HbR}$ during the acute phase were captured with high temporal and spatial resolution through the intact skull. Hemodynamic changes that were correlated with vasoconstrictive events, peri-infarct spreading depressions (PISD), were observed. These experiments demonstrate the utility of LSF and Multi-spectral reflectance imaging (MSRI) in mouse disease models.
\end{abstract}

\section{INTRODUCTION}

Recent advances in speckle contrast imaging [1, 2], and multispectral reflectance imaging [3] have made it possible to image changes in oxyhemoglobin $(\mathrm{HbO})$ and deoxyhemoglobin $(\mathrm{HbR})$ concentrations, blood volume (total hemoglobin, $\mathrm{HbT}$ ), and blood velocity $(\mathrm{CBF})$, in an intact, anesthetized animal. The advantage of simultaneous $\mathrm{CBF}, \mathrm{HbT}$ and oxygenation measurement, is the ability to extract changes in cerebral metabolic rate of oxygen consumption $\left(\mathrm{CMRO}_{2}\right)[4]$ from the fractional changes in $\mathrm{CBF}, \mathrm{HbR}$, and $\mathrm{HbT}$;

$$
C M R O_{2}=\left(1+\frac{\Delta C B F}{C B F_{0}}\right)\left(1+\gamma_{R} \frac{\Delta H b R}{H b R_{0}}\right)\left(1+\gamma_{T} \frac{\Delta H b T}{H b T_{0}}\right)^{-1}
$$

The most common application of these techniques is the study of functional activation [5,6]. Small changes in hemoglobin concentrations can be estimated using a modified Beer-Lambert law [7]. Which is is not expected to be valid for large changes in chromaphore.

In the current study we develop a method to acquire MSRI and LSF images simultaneously from mouse brain in the experimental setting of ischemia. We introduce a new method for analysis of multispectral data. The combined techniques allow simultaneous monitoring of $\mathrm{HbO}, \mathrm{HbR}$ and $\mathrm{CBF}$ at the same spatial locations, allowing for the calculation of $\mathrm{CMRO}_{2}$ timecourses during ischemia at multiple locations with respect to the ischemic core, penumbra and non-ischemic cortex $[8,9]$. The technique allows us to monitor these hemodynamic parameters during distal occlusion of the middle cerebral artery, the subsequent anoxic depolarization (AD) and peri-infarct spreading depolarizations (PIDs) with high spatial and temporal resolution.

\section{METHODS}

\subsection{General Surgical Preparations}

Mice (C57BL/6J, 25-35g) were anesthetized with isofluorane (2\% induction and $1 \%$ maintenance), endothracheally intubated, and ventilated (70 \% $\mathrm{N}_{2} \mathrm{O} 30 \% \mathrm{O}_{2}$; SAR 830/P, CWE, Ardmore, PA). The femoral artery was cannulated for the purposes of monitoring blood pressure (BP) (ETH-400 transducer amplifier, ADInstruments, MA). 

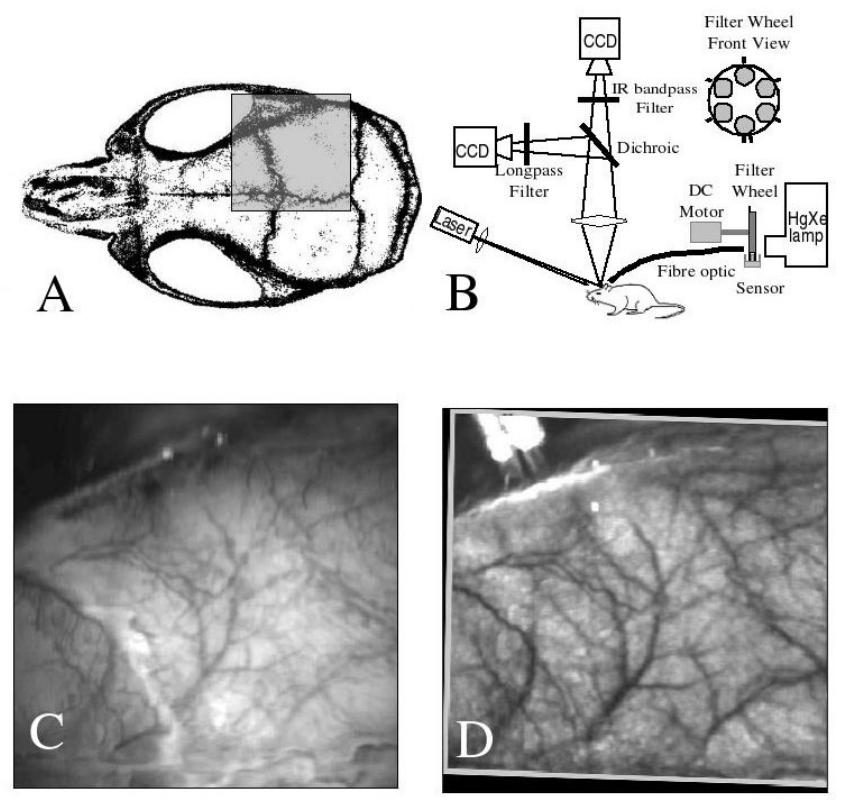

Figure 1. Combined multispectral and speckle contrast imaging of mouse cortex. A representation of the mouse skull is shown in Panel A; the gray square illustrates the area that is imaged. A schematic of the system setup is shown in panel B. Panels $\mathrm{C}$ and D show a 580nm reflectance image and a speckle contrast image, respectively.

$\mathrm{BP}$ and heart rate were continuously monitored and recorded (PowerLab, ADInstruments, MA). The femoral vein on the opposite side was similarly cannulated for use in intravenous drug administration. Mice were paralyzed (pancuronium, $0.4 \mathrm{mg} / \mathrm{kg} / \mathrm{h}$ i.p.) and placed on a sterotaxic frame. The scalp and peristeum were pulled aside and mineral oil was applied to the exposed skull to prevent drying. Adequacy of the anesthesia was checked regularly by the absence of a blood pressure response to tail pinch. Body temperature was maintained at $37^{\circ} \mathrm{C}$ using a thermostatic heating pad (FHC, Brunswick, ME). Arterial blood gases and $\mathrm{pH}$ were measured at least once per hour using $30 \mu \mathrm{L}$ samples (Blood Gas Analyzer 248, CIBA/Corning, NY) and used to maintain all physiological parameters within previously reported normal limits. Institutional guidelines for the care and use of animals were strictly followed and all animal protocols were approved by the MGH center for comparative medicine.

\subsection{Focal cerebral ischemia}

For focal cerebral ischemia, after general surgical preparation, the temporalis muscle was separated from the temporal bone and excised. A burr hold (2mm diameter) was drilled through the temporal bone, just above the zygomatic arch, whilst saline was applied as a coolant. The dura was kept intact and a microvascular clip (Ohwa Tsusho, Tokyo, Japan) was used to occlude the distal middle cerebral artery (dMCA).

\subsection{Optical imaging}

Figure 1 is a schematic illustration of the instrument used in these experiments. The instrument is similar to one used in previously published studies [10], with a few important differences. Light for multi-spectral imaging is provided by a halogen fiberoptic illuminator (Techniquip R150, Capra Optical, Natick, MA) and passes through $10 \mathrm{~nm}$ wide bandpass filters, ranging from $560 \mathrm{~nm}$ to $610 \mathrm{~nm}$. As in the previously described instrument, a laser diode $(785 \mathrm{~nm}, 70 \mathrm{~mW})$ provides the coherent illumination required for LSF imaging. The light is captured using a variable magnification objective $(\times 0.75$ to $\times 3$, Edmund Optics, Barrington, NJ) and focused either through (IR laser) or reflected off of (Visible light), a dichroic mirror onto two CCD cameras (Coolsnap fx, Roper Scientific $343 \times 434$ pixels, for MSRI; Cohu 4600, San Diego, CA, $640 \times 480$ pixels, for LSF). Raw MSRI data was collected in sequences of 30 frames at $10 \mathrm{~Hz}$, with one sequence approximately every 7 seconds. The reflectance image 
from each wavelength was averaged over the sequence. Raw speckle data was similarly collected in sequences of 10 frames at $15 \mathrm{~Hz}$ every 7 seconds and converted into speckle contrast images before averaging.

\subsection{Image analysis}

The details of CBF imaging using speckle contrast have been published previously [3]. Simply put, a speckle contrast image is calculated by dividing the standard deviation by the average intensity $\sigma /\langle I\rangle$ in a small region $(7 \times 7$ pixels). The speckle contrast is inversely dependent on the CBF [11]. Here, we will focus on the method by which $\mathrm{HbO}$ and $\mathrm{HbR}$ are extracted from MSRI.

The multispectral analysis used in our previous work follows the work of Kohl et al [7]. Beer's law states that the intensity of light passing through an absorbing media falls off exponentially as the fraction of light absorbed during each unit path length is constant. In a turbid media, like tissue, the path length is highly dependent on chromaphore concentrations and scattering, which in turn is wavelength dependent. Monte-Carlo simulations of photon path lengths at an assumed baseline concentration can be conducted [12]. In a simplyfied form, the change in attenuation $\left(\Delta A=\log _{10}\left(R / R_{0}\right), \mathrm{R}=\right.$ reflected light intensity), can be given by [13]:

$$
\Delta A(\lambda)=\sum_{i=1}^{N_{\text {photons }}}-\frac{\mu_{a}(\lambda) L_{i}\left(\mu_{a}, \mu_{s}^{\prime}\right)}{N}
$$

where $\sum_{i=1}^{N_{\text {photons }}} L_{i}\left(\mu_{a}, \mu_{s}^{\prime}\right) / N$ is the average path length and $\mu_{a}(\lambda)=\sum_{j=1}^{N_{\text {chromo }}} \epsilon_{j}(\lambda) \Delta c_{j}$, is the absorption coefficient. $N_{\text {chromo }}$ is the number of chromophores, $\epsilon_{k}(\lambda)$ is the molar exctinction coefficient, and $c_{j}$ is the concentration. Linearization is achieved by introducing a differential path length factor $\left(D_{\alpha}\right)[14]$, which assumes that differential absorption due to path length changes are linearly dependent on changes in chromophore concentrations. Under this assumption, equation 2 becomes:

$$
\Delta A(\lambda)=\sum_{k=1}^{N_{\text {chromo }}} \epsilon_{k}(\lambda) \Delta c_{k} D_{\alpha}(\lambda)
$$

Kohl et al also consider that, in principle, scattering changes can be accounted for, or calculated by, considering the change in attenuation due to scattering loss as a pseudo-chromophore; they introduce a scattering differential path length factor and scattering pseudo-concentration.

\section{RESULTS}

\subsection{Modified Beer-Lambert approximation fails if $\mathrm{HbO}$ is reduced by more than $\approx 20 \%$.}

The differential path length factor, as used in the modified Beer-Lambert law, involves a first order approximation to the change in attenuation due to the change in pathlength. The approximation will hold only if the changes in attenuation due to changes in path length is linearly dependent on chromophore concentration. Since changes in chromophore concentration are large during ischemia, numerical analysis of the discrepancy between a non-linear fit to equation 2 and the modified Beer-Lambert approximation (equation 3) was conducted.

Comparison of the analysis of a typical timecourse for a mouse undergoing dMCAO using the modified Beer-Lambert approximation and the non-linear fitting method shows that the modified Beer-Lambert law gives changes in concentration that are physically impossible, whereas the least squares fitting method does not. At 50 minutes after occlusion the value of $1+\Delta \mathrm{HbO} / \mathrm{HbO}$ over a region of interest inside the ischemic core, for the modified Beer-Lambert law is -0.5085 , inclusion of scattering as a pseudo-chromophore increases the negative value further to -0.5348 . Applying the least squares technique gives values of 0.1966 and 0.1689 with scattering fixed at $150 \mathrm{~cm}^{-1}$ and allowed to be fit, respectively. Changes in HbR over the same timecourse do not become physically impossible but do show large differences between the analysis techniques. The modified Beer-Lambert approximation returns values of 1.5217 without scattering and 1.4215 with scattering whereas the non-linear solution yields values of 1.1795 and 1.1258. 

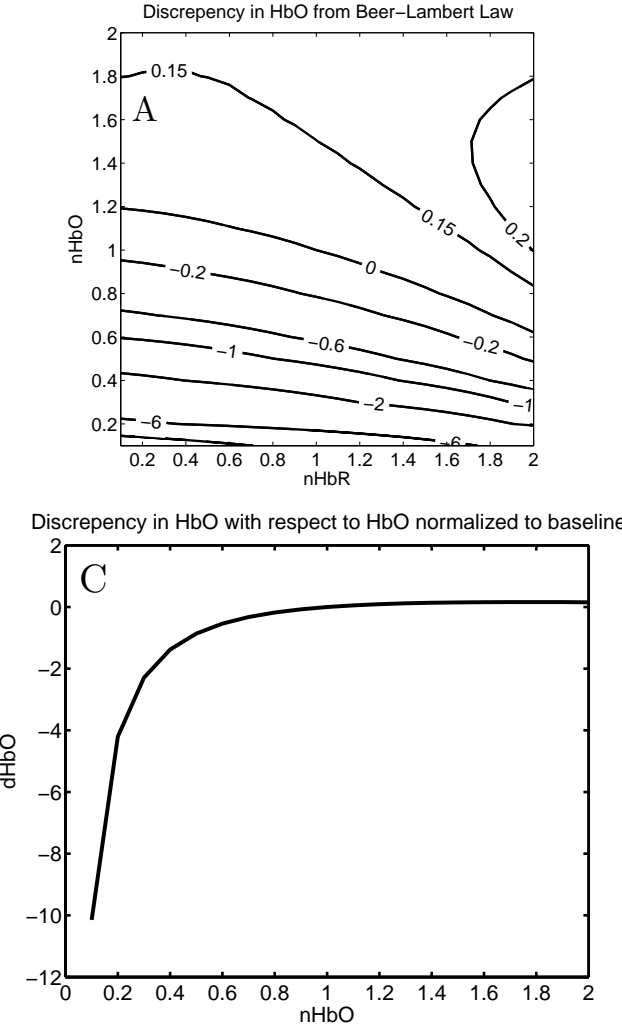
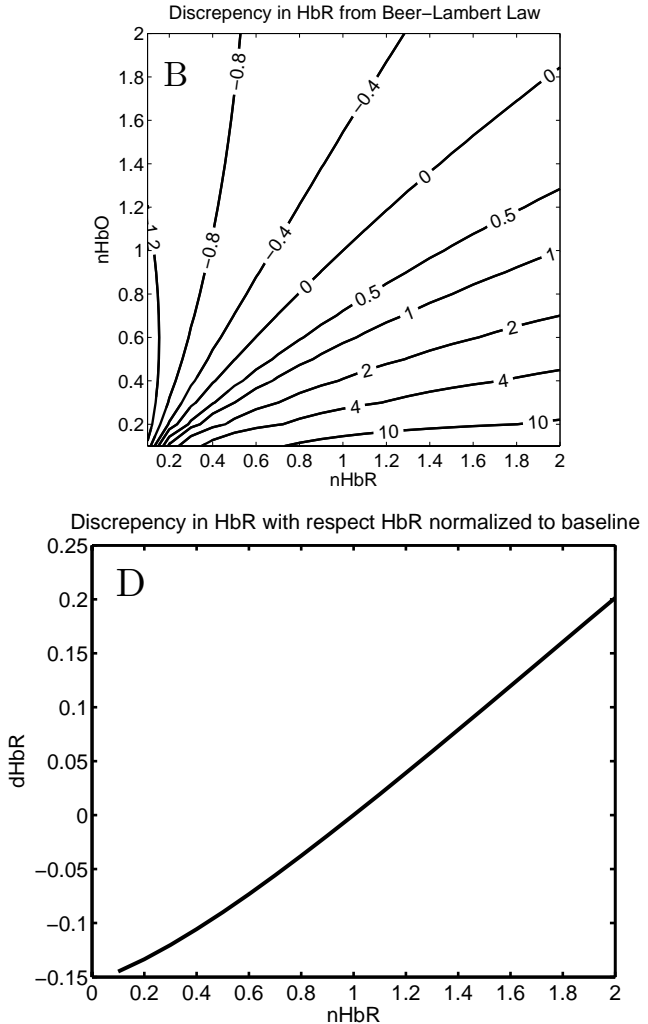

Figure 2. Discrepancy between non-linear fit and the modified Beer-Lambert Approximation. Baseline chromophore concentrations of $\mathrm{HbO}=100$ and $\mathrm{HbR}=60 \mu \mathrm{gml}^{-1}$ were assumed and expected changes in reflectance in the six wavelengths used were calculated. Those predicted changes were used to calculate the predicted chromophore concentrations using the modified Beer-Lambert law which were then normalized by the simulated changes. The result is the fractional discrepancy caused by the Beer-Lambert linearization with respect to change in chromophore concentrations.

The large discrepancy between the two methods, and relatively small difference made by adding the scattering corrections implies that the major source of systematic error in this circumstance is the linear modified BeerLambert approximation and not the scattering correction. To that end, simulated changes in chromophore concentrations from the assumed baseline of $\mathrm{HbO}=100 \mu \mathrm{g} / \mathrm{ml}$ and $\mathrm{HbR}=60 \mu \mathrm{g} / \mathrm{ml}$ were used to calculate expected changes in reflectance for the 6 wavelengths used $(560 \mathrm{~nm}-610 \mathrm{~nm}$ in $10 \mathrm{~nm}$ intervals) using equation 2. The calculated changes in reflectance were used to calculate chromophore concentrations using the modified Beer-Lambert method. The results from these simulations give the discrepancy for both chromophores caused by the linearizion, with respect to changes in each chromophore.

Figure 2 shows the discrepancy in the calculated values, $\left(\left\{d C=C_{\text {calculated }} / C_{\text {simulated }}\right\}-1\right)$, against $n C=$ $C_{\text {simulated }} / C_{0}$, where $C=\mathrm{HbO}$ or $\mathrm{HbR}$. Panel A shows that, except for large $\Delta \mathrm{HbR}$ with small $\Delta \mathrm{HbO}$, the modified Beer-Lambert Law overestimates $\Delta \mathrm{HbO}$ over the choice of wavelengths used. This is shown by the fact that for $\mathrm{nHbO}>1$, the $\mathrm{dHbO}$ is generally greater than zero and vice-versa. Panel $\mathrm{B}$ shows that, generally if $\mathrm{nHbR}>\mathrm{nHbO}$, which means that blood oxygenation is reduced as a result of the change, then HbR will be overestimated but if the reverse is true, $\mathrm{HbR}$ will be underestimated. The degree of the discrepancy lessens with increased blood volume, as shown by the contour lines diverging at higher values of $\mathrm{HbR}$ and $\mathrm{HbO}$.

Panels C and D show how the discrepancy in the chromophore would vary with respect to chromophore concentration if only one chromophore were varied. Although this is an unrealistic assumption, Panel $\mathrm{C}$ shows that $\mathrm{dHbO}$ becomes negative and large with only modest changes in HbO. The discrepancy in HbR is serious, but there is no catastrophic breakdown, like the one seen when $\mathrm{HbO}$ is varied. These results are consistent with the observations made at the beginning of this section. 
The disadvantage of the non-linear fitting technique is that for each new combination of parameters called by the fitting routine, the absorption over pathlengths generated by the Monte-Carlo simulations must be recalculated. When the scattering correction is included, a new Monte-Carlo simulation must be used. To accommodate this requirement, Monte-Carlo simulations for a range of scattering coefficients $\left(100-200 \mathrm{~cm}^{-1}\right.$ at $5 \mathrm{~cm}^{-1}$ intervals) were pre-computed and the precise value of $\mu_{a}$ calculated by interpolating between the two nearest values.

Qualitative comparison of the timecourses for the ischemic core, penumbra, and non-ischemic cortex (not shown) reveals that the two sets of solutions are qualitatively similar and so spatial-temporal maps generated by the modified Beer-Lambert approximation may still be used to identify the ischemic core, penumbra and non-ischemic cortex for use in setting regions of interest for generation of timecourses.

\subsection{Hemodynamic timecourses immediately following dMCA occlusion}

Timecourses of the various hemodynamic parameters are shown in Figure 3. Upon dMCAO, CBF drops to $\approx 30 \%$ of baseline levels in the ischemic core. No change in HbT is observed for the first minute or so, although the level of $\mathrm{HbO}$ decreases and $\mathrm{HbR}$ increases, as expected due to the longer blood transit time. A similar effect is observed in the penumbra whilst in the non-ischemic cortex, there are no changes in any of the hemodynamic parameters.

After a time interval of the order of a minute, a further reduction in CBF is accompanied by a large reduction in $\mathrm{HbO}$, and $\mathrm{HbR}$ in the core. This effect is taken to be representative of anoxic depolarization (AD), which leads to peri-infarct spreading depression(PID). Both AD and PIDs are electophysiologically similar to cortical spreading depression (CSD). In the penumbra, the transient effect of the $\mathrm{AD}$ on $\mathrm{HbO}$ is more pronounced with a dip to below $30 \%$ at around the 2 minute mark. Afterward, CBV recovers slightly but is not restored to pre-ischemic values.

In the non-ischemic cortex, the first PID causes a decrease in HbT, flow and metabolism followed by a partial recovery. This recovery could be interpreted as an increase in oxygen demand caused by the additional metabolic burden of the PID in tissue that is still perfused. Despite this extra metabolic demand, HbT does not completely recover in the non-ischemic cortex in the mouse.

\section{3. $\mathrm{CMRO}_{2}$ timecourses during peri-infarct spreading depressions}

Figure 4 shows the $\mathrm{CMRO}_{2}$ timecourses for an ischemic mouse during a series of four PIDs. It has been presumed that PIDs increase the metabolic burden on tissue due to the massive redistribution of ions across membranes and the extra energy requirement for repolarization [2]. Indeed in the non-Ischemic region of the cortex, the initial drop in $\mathrm{CMRO}_{2}$ associated with the PID is followed by an overshoot. We hypothesize that this overshoot represents endogenous neuro-vascular coupling.

The $\mathrm{CMRO}_{2}$ timecourse taken from the ischemic core shows a drop in $\mathrm{CMRO}_{2}$ but no reactive overshoot. The drop in $\mathrm{CMRO}_{2}$ verifies that oxygen consumption is still taking place between PIDs in the core whilst the absence of a hyperemic response indicates that neurovascular coupling is inhibited in the ischemic core. We hypothesize that this lack of a hyperemic response is a clear indication that metabolism is delivery limited in the core.

\section{DISCUSSION}

There has been much interest in the measurement of $\mathrm{CMRO}_{2}$ in the ischemic and injured brain in both animal models [15] and human patients [16, 17]. Metabolic measures are thought to be a strong indicator of tissue outcome [18] with oxygen and glucose metabolism being closely linked [19]. LSF imaging has previously been applied to cerebral ischemia [2] and it's advantages over laser Doppler flowmetry have been established. MSRI, which allows for the imaging and separation of both hemoglobins has not previously been demonstrated in a stroke model. This study represents the first successful attempt to monitor $\mathrm{CMRO}_{2}$ in vivo in an intact mouse during mild to moderate cerebral focal ischemia.

MSRI is widely used to assess changes in cortical concentrations of oxy- and deoxyhemoglobin from changes in light reflectivity as predicted by the Beer-Lambert law. Recently, the work of Kohl [7] explored the use of 


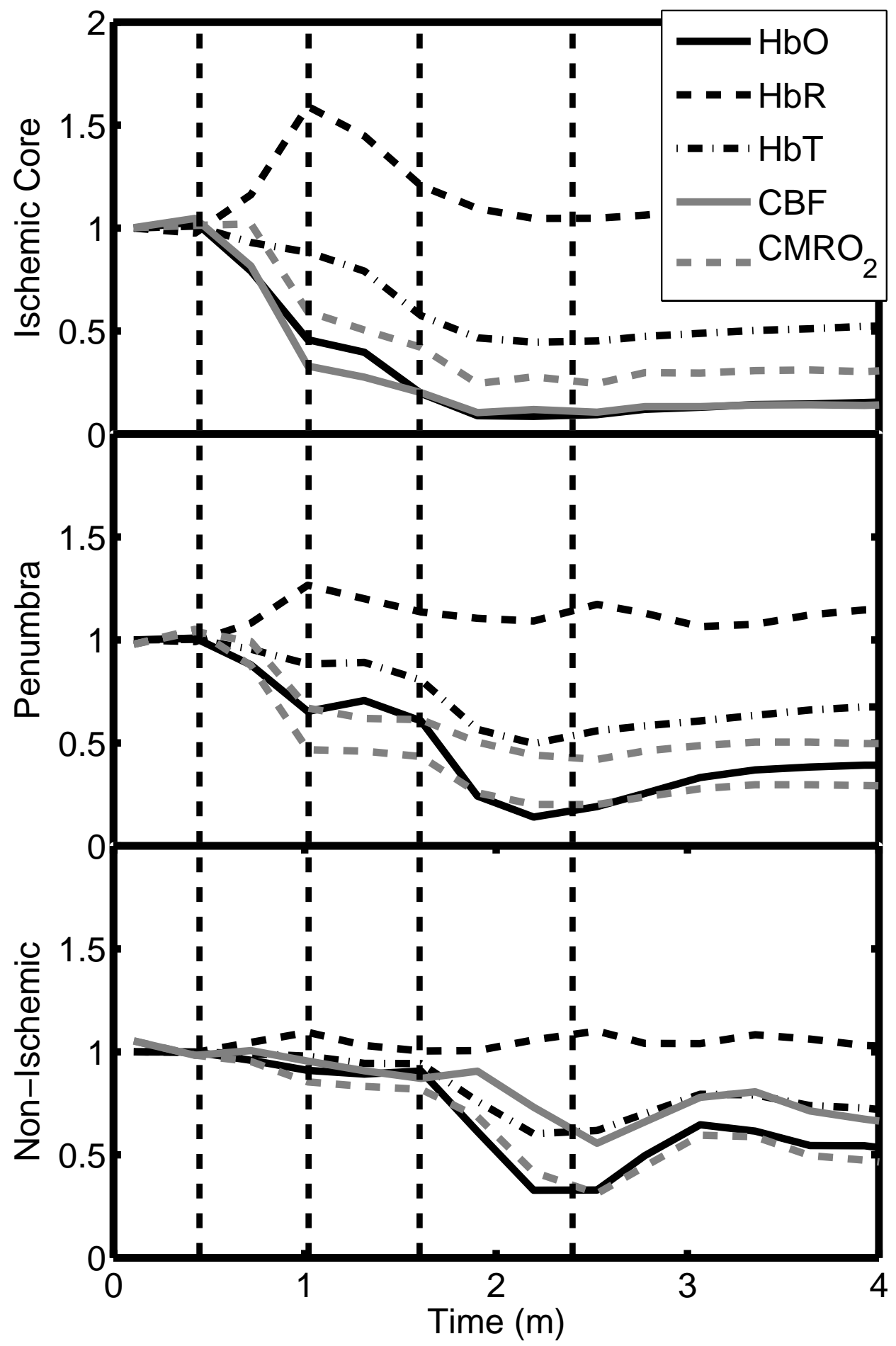

Figure 3. Timecourses of hemodynamic parameters during dMCAO and subsequent anoxic depolerization.Drop in $\mathrm{CMRO}_{2}$ occurs as a two stage process. The initial phase is a direct result of reduced CBF from the occlusion. The second drop in $\mathrm{HbO}$, and $\mathrm{CBF} \mathrm{CMRO}_{2}$, in the core and penumbra are associated with the first PID, associated with AD. Reduced metabolism in the non-ischemic cortex follows the PID. 


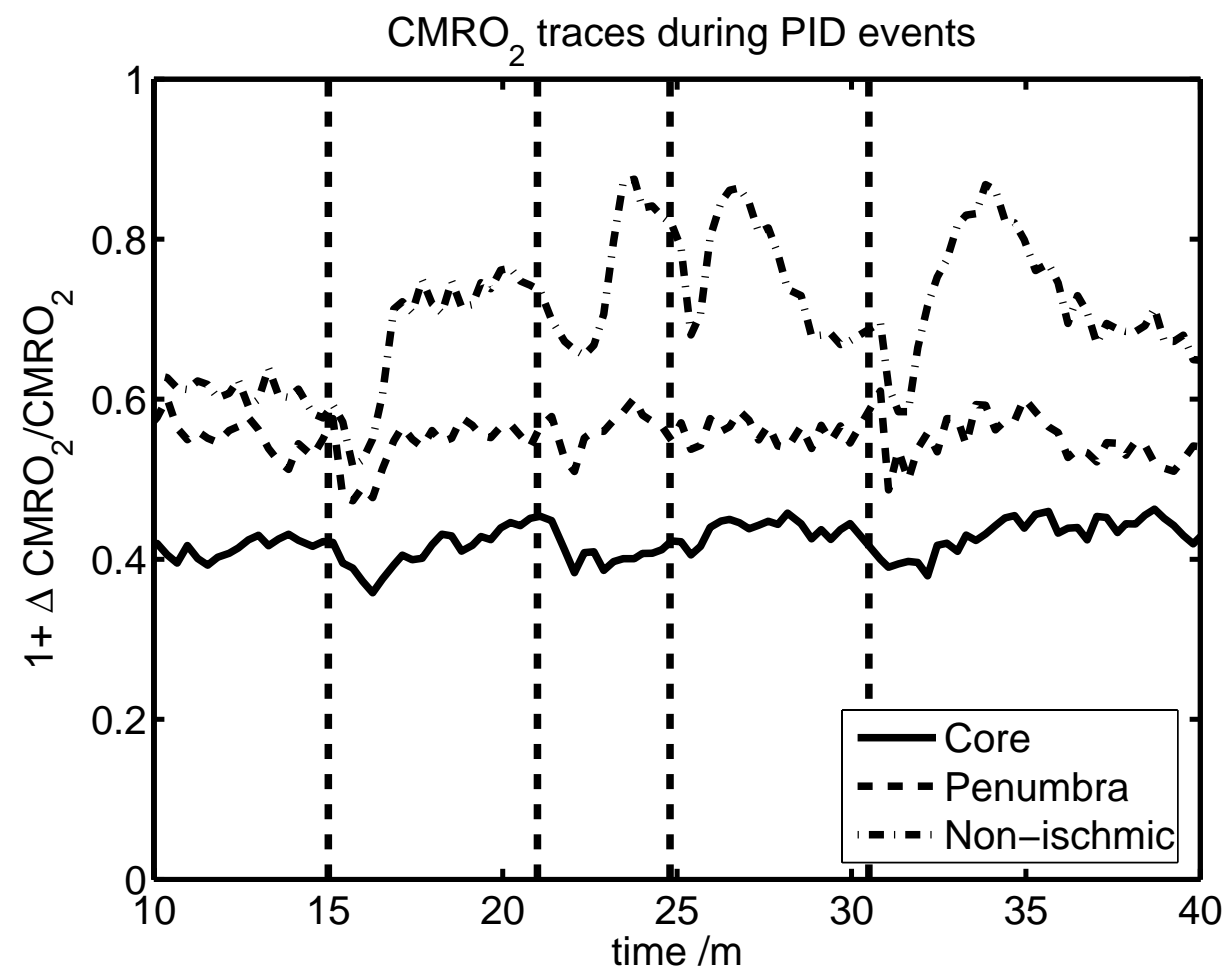

Figure 4. Timecourses of the effects of four consecutive PIDs on the ischemic core, penumbra and non-ischemic cortex.

the modified Beer-Lambert approximation in intrinsic optical imaging. The authors made use of the differential path length factor $D_{a}$ [14], which represents the first term in a Taylor expansion of the change in absorption due to change in scattering:

$$
\Delta A(\lambda)=\frac{\partial A}{\partial \mu_{a}} \Delta \mu_{a}+\frac{\partial^{2} A}{\left(\partial \mu_{a}\right)^{2}} \frac{\left(\Delta \mu_{a}\right)^{2}}{2}+\ldots
$$

where only the first term is not ignored. Upon analysing the multi-spectral data from these experiments, it was found that the approximation does not hold well under large changes, especially in the case of a reduction in $\mathrm{HbO}$, and so an alternative method of analysis is proposed for this case. Here, for each time-point of a region of interest, equation 2 is non-linearly fitted to the changes in reflectance. The disadvantage of this technique being the drastic increase in time taken to non-linearly fit each data point. In the future, we wish to develop an alternative form of regularization in order to accelerate the analysis and make fully quantified spatio-temporal maps of $\mathrm{CMRO}_{2}$ in the ischemic mouse brain.

PIDs and AD are electrophysiologically similar to cortical spreading depression (CSD) [20]. A slow wave of negative potential shift propagates at 2 to $4 \mathrm{~mm} / \mathrm{min}$ over the cerebral cortex. Extracellular $\mathrm{K}^{+}$increases to 30 to $60 \mathrm{mM}$, along with elevations in extracellular levels of excitatory neurotransmitters. The presence of PIDs, following cerebral ischemia has been previously documented in human patients after head injury [21] and a role in the pathophysiology of ischemia has been suggested [22], [23]. It is hypothesized that the ischemic brain cannot increase CBF to match the increased metabolic demand caused by repolarization, thereby exacerbating the oxygen deficit and worsening the ischemic injury [24].

Here, we demonstrate measurement of $\mathrm{CMRO}_{2}$ during initial $\mathrm{AD}$ and subsequent PIDs over regions of interest in three pathologically important regions of the cortex in relation to stroke. We have found that at the point of dMCAO, CBF dramatically decreases but that there is little to no change in HbT, even in the core, during 
this initial phase. An increase in $\mathrm{HbR}$ and a decrease in $\mathrm{HbO}$ is observed as the slower moving blood spends more time in the capillary bed and de-oxygenated. In the non-ischemic cortex, there was little effect on any hemodynamic parameter. After a minute or so, $\mathrm{HbT}$ decreases, suggesting either passive vascular collapse or active vasoconstriction. This event is accompanied by a further reduction in $\mathrm{CBF}$ and reductions in $\mathrm{HbT}$ and $\mathrm{CMRO}_{2}$ in the non-ischemic cortex. We hypothesize that this event is triggered by the developing hypoxia in the $\mathrm{AD}$.

During subsequent PIDs, $\mathrm{CMRO}_{2}$ was reduced in each of the three regions of interest. Following this initial dip, there is a $\mathrm{CMRO}_{2}$ overshoot in the non-ischemic cortex that does not occur in the core. This overshoot may be a highly useful endogenous measure of neuro-vascular coupling. The drop in $\mathrm{CMRO}_{2}$ in the core and the lack of a $\mathrm{CMRO}_{2}$ overshoot suggest that oxygen consumption in the core is delivery limited.

\section{CONCLUSION}

Combined MSRI and LSF imaging allows for simultaneous monitoring of $\mathrm{HbO}, \mathrm{HbR}, \mathrm{CBF}$, and $\mathrm{CMRO}_{2}$ with high spatial and temporal resolution, in the mouse cortex during dMCAO; a commonly applied model of focal cerebral ischemia. The generally applied modified Beer-Lambert approximation is not valid for large changes in chromophore and so a non-linear fitting routine that makes use of pathlengths generated from Monte-Carlo simulations can be used to give quantified time-courses of $\mathrm{HbO}$ and $\mathrm{HbR}$.

During cerebral ischemia, we note that $\mathrm{AD}$ follows occlusion of the MCA by about a minute and leads to a reduction in $\mathrm{CBF}, \mathrm{HbT}$, and $\mathrm{CMRO}_{2}$ in the non-ischemic cortex. Furthermore, endogenous neurovascular coupling due to PIDs is inhibited in the ischemic core, indicating supply limited metabolism whilst coupling appears to be intact in the non-ischemic cortex.

\section{References}

[1] B. Ruth, "Blood flow determination by the laser speckle method," Int J Microcirc Clin Exp 9(1), pp. 21-45, 1990. 0167-6865 Journal Article.

[2] C. Ayata, A. K. Dunn, O. Y. Gursoy, Z. Huang, D. A. Boas, and M. A. Moskowitz, "Laser speckle flowmetry for the study of cerebrovascular physiology in normal and ischemic mouse cortex," J Cereb Blood Flow Metab 24(7), pp. 744-55, 2004. 0271-678x Journal Article.

[3] A. K. Dunn, H. Bolay, M. A. Moskowitz, and D. A. Boas, "Dynamic imaging of cerebral blood flow using laser speckle," J Cereb Blood Flow Metab 21(3), pp. 195-201, 2001. 0271-678x Journal Article.

[4] M. Jones, J. Berwick, and J. Mayhew, "Changes in blood flow, oxygenation, and volume following extended stimulation of rodent barrel cortex," Neuroimage 15(3), pp. 474-87, 2002. 1053-8119 Journal Article.

[5] A. Villringer and B. Chance, "Non-invasive optical spectroscopy and imaging of human brain function," Trends Neurosci 20(10), pp. 435-42, 1997. 0166-2236 Journal Article Review Review, Tutorial.

[6] J. Mayhew, Y. Zheng, Y. Hou, B. Vuksanovic, J. Berwick, S. Askew, and P. Coffey, "Spectroscopic analysis of changes in remitted illumination: the response to increased neural activity in brain," Neuroimage 10(3 Pt 1), pp. 304-26, 1999. 1053-8119 Journal Article.

[7] M. Kohl, U. Lindauer, G. Royl, M. Kuhl, L. Gold, A. Villringer, and U. Dirnagl, "Physical model for the spectroscopic analysis of cortical intrinsic optical signals," Phys Med Biol 45(12), pp. 3749-64, 2000. 0031-9155 Journal Article.

[8] S. M. Davis and G. A. Donnan, "Advances in penumbra imaging with mr," Cerebrovasc Dis 17 Suppl 3, pp. 23-7, 2004. 1015-9770 Journal Article Review Review, Tutorial.

[9] M. Fisher, "The ischemic penumbra: identification, evolution and treatment concepts," Cerebrovasc Dis $\mathbf{1 7}$ Suppl 1, pp. 1-6, 2004. 1015-9770 Journal Article Review. 
[10] A. K. Dunn, A. Devor, H. Bolay, M. L. Andermann, M. A. Moskowitz, A. M. Dale, and D. A. Boas, "Simultaneous imaging of total cerebral hemoglobin concentration, oxygenation, and blood flow during functional activation," Opt Lett 28(1), pp. 28-30, 2003. 0146-9592 Journal Article.

[11] J. D. Briers, "Laser doppler, speckle and related techniques for blood perfusion mapping and imaging," Physiol Meas 22(4), pp. R35-66, 2001. 0967-3334 Journal Article Review.

[12] K. H. Hayakawa, J. Spanier, F. Bevilacqua, A. K. Dunn, J. S. You, B. J. Tromberg, and V. Venugopalan, "Perturbation monte carlo methods to solve inverse photon migration problems in heterogeneous tissues.," Opt Lett 26(17), pp. 1335 - 1337, 2001.

[13] D. A. Boas, J. P. Culver, J. J. Stott, and A. K. Dunn, "Three dimensional monte carlo code for photon migration through complex heterogeneous media including the adult human head.," Opt Express 10(3), pp. 159-170, 2002.

[14] S. R. Arridge, M. Cope, and D. T. Delpy, "The theoretical basis for the determination of optical pathlengths in tissue: temporal and frequency analysis," Phys Med Biol 37(7), pp. 1531-60, 1992. 0031-9155 Journal Article.

[15] N. C. Singh, P. M. Kochanek, J. K. Schiding, J. A. Melick, and E. M. Nemoto, "Uncoupled cerebral blood flow and metabolism after severe global ischemia in rats," J Cereb Blood Flow Metab 12(5), pp. 802-8, 1992. 0271-678x Journal Article.

[16] P. Vespa, M. Bergsneider, N. Hattori, H. M. Wu, S. C. Huang, N. A. Martin, T. C. Glenn, D. L. McArthur, and D. A. Hovda, "Metabolic crisis without brain ischemia is common after traumatic brain injury: a combined microdialysis and positron emission tomography study," J Cereb Blood Flow Metab 25(6), pp. 76374, 2005. 0271-678x Clinical Trial Journal Article.

[17] G. J. Bouma and J. P. Muizelaar, "Cerebral blood flow in severe clinical head injury," New Horiz 3(3), pp. 384-94, 1995. 1063-7389 Journal Article Review Review, Tutorial.

[18] P. Frykholm, J. L. Andersson, J. Valtysson, H. C. Silander, L. Hillered, L. Persson, Y. Olsson, W. R. Yu, G. Westerberg, Y. Watanabe, B. Langstrom, and P. Enblad, "A metabolic threshold of irreversible ischemia demonstrated by pet in a middle cerebral artery occlusion-reperfusion primate model," Acta Neurol Scand 102(1), pp. 18-26, 2000. 0001-6314 Journal Article.

[19] P. Frykholm, L. Hillered, B. Langstrom, L. Persson, J. Valtysson, and P. Enblad, "Relationship between cerebral blood flow and oxygen metabolism, and extracellular glucose and lactate concentrations during middle cerebral artery occlusion and reperfusion: a microdialysis and positron emission tomography study in nonhuman primates," J Neurosurg 102(6), pp. 1076-84, 2005. 0022-3085 Journal Article.

[20] G. G. Somjen, "Mechanisms of spreading depression and hypoxic spreading depression-like depolarization," Physiol Rev 81(3), pp. 1065-96, 2001. 0031-9333 Journal Article Review.

[21] A. J. Strong, M. Fabricius, M. G. Boutelle, S. J. Hibbins, S. E. Hopwood, R. Jones, M. C. Parkin, and M. Lauritzen, "Spreading and synchronous depressions of cortical activity in acutely injured human brain," Stroke 33(12), pp. 2738-43, 2002. 1524-4628 Clinical Trial Journal Article.

[22] N. M. Branston, A. J. Strong, and L. Symon, "Extracellular potassium activity, evoked potential and tissue blood flow. relationships during progressive ischaemia in baboon cerebral cortex," J Neurol Sci 32(3), pp. 305-21, 1977. 0022-510x Journal Article.

[23] T. Back, M. D. Ginsberg, W. D. Dietrich, and B. D. Watson, "Induction of spreading depression in the ischemic hemisphere following experimental middle cerebral artery occlusion: effect on infarct morphology," J Cereb Blood Flow Metab 16(2), pp. 202-13, 1996. 0271-678x Journal Article.

[24] C. Ayata, H. K. Shin, S. Salomone, Y. Ozdemir-Gursoy, D. A. Boas, A. K. Dunn, and M. A. Moskowitz, "Pronounced hypoperfusion during spreading depression in mouse cortex," J Cereb Blood Flow Metab 24(10), pp. 1172-82, 2004. 0271-678x Journal Article. 